\section{BLOOD EXAMINATION.}

ITS VALUE TO THE GENERAL PRACTITIONER.*

\section{H. FLSSELL, M.D.}

PIIILADEIPHIA.

I believe that the vast majority of the practitioners of medicine never make an examination of the blood of their patients unless such an examination is suggested and carried out by a consultant. The reasons for this may be many, but the following I believe to be the most important: 1. Blood examinations as practical diagnostic measures are of comparatively recent origin; consequently, men who graduated fifteen or more years ago were not taught the value of such examinations, or how to make them. Unless such members of our profession have had the subject forced upon them by teaching, or by continued intercourse with men trained in such work, they are quite unlikely to take it upon themselves. 2. Blood examinations, like all other valuable diagnostic measures, take time. The general practitioner is hurried;in the town competition is great; and those who depend upon the practice of medicine for a livelihood are apt to become careless about the use of these finer diagnostic measures, and depend upon less certain means of diagnosis. To once slur these valuable agents is to learn to gradually discard them, to the great detriment of ourselves and our patients. It is certain that in many cases examination of the blood is quite as important as, indeed more important than, examination of the urine. It is now an accepted fact that no case is thoroughly studied without a urine examination. I believe it will soon be accepted by the general practitioner - as it is now recognized by the specialist-that a blood examination is equally asimportantfor a thorough study of our cases.

That such examinations are possible and practical for one who practices medicine for a livelihood and not as a pastime, $I$ am personally certain, for $I$ am a practitioner and by no means a hematologist; I have been slow in learning, but now make almost daily use of this most valuable help. Without the aid of an assistant, I have made examinations of blood in a practical way just as I have the urine, and I feel that it helps me as much in my daily work. I have as yet been unable to examine the blood of every patient, but I trust that the time is not far off when I can do that in my work. I do, however, use blood examinations in every obscure case, with the greatest possible benefit. I was led to the general use of examination of the blood by the recognition of my own mistakes and by the mistakes of others where no such examinations were made.

One of the most common errors is that of considering all pale individuals as anemic. Many mill-workers, or workmen who have but little daylight work in the open air are extremely white, but far from anemic. The cheeks may be ever so pale, the hands white, but the lips and conjunctivæ fail to show lack of coloring matter in the blood. Without an accurate blood examination such individuals are frequently given iron for long periods of time, a proceeding which is certainly useless and often harmful.

One of the most striking cases of this sort presented itself at my dispensary at the University of Pennsylvania last winter-a man, aged 32 , a carpenter, presented himself with symptoms of general weakness with some digestive disturbances, but without loss of flesh. His skin

*Presented to the Section on Practice of Medicine, at the Fifty-first Annual Meeting of the American Medical Association, held at Atlantic City, N. J., June 5-8, 1900. was extremely pale, but repeated examinations showed his hemoglobin to be 90 per cent., with a normal number of red corpuscles. Certainly any one, without most careful observation or without a blood-count, would have considered this man anemic and have uselessly given iron. The examination of his blood excluded any form of anemia.

The opposite condition of affairs is just as frequently present. A person may have at the time of examination a flushed face and give every indication, as far as the skin is concerned, of a normal blood-count, yet examination will show a decided reduction of the hemoglobin. An example of this condition presented itself in my office while this paper was in process of preparation. A girl of 17 was brought by Dr. Evans, of Philadelphia. She had been told a number of years ago, that she was likely to die of aneurysm of the arch of the aorta. She was extremely nervous, and presented all the symptoms of a neurasthenic. Physical examination at once disposed of the question of aneurysm. Her face was florid, unusually red for one who was ill; her lips, however, suggested that she might be lacking in some of her blood elements. Examination for hemoglobin showed only 65 per cent., notwithstanding her florid appearance.

Perhaps no class of patients are treated with less foresight or with less accuracy than arechlorotic girls. They present themselves to their physicians with symptoms such as these: dyspnea, palpitation of the heart, perhaps edema, or hemorrhage from some mucous membrane. Probably a slight cough accompanies the disorder, together with loss of appetite and rapidly increasing pallor. Time and again such individuals have presented themselves at our clinic with a diagnosis of tuberculosis or of heart disease. An accompanying bronchitis,or the rapidly beating heart with hemic murmurs have lent color to this diagnosis. An examination of the blood with the proper instruments, which is done very quickly, shows the hemoglobin to be from 20 to 50 per cent., with but little reduction in the number of red corpuscles. The physical signs then become explicable, and what was a hopeless case becomes one amenable to treatment. Such a mistake in diagnosis occurred in my own practice. In my first year I was called to see a girl of 16 years; she was pale, and had a general edema; her heart's action was tumultuous, and a loud systolic murmur was heard over the entire organ. I made a diagnosis of organic heart disease, but fortunately put the patient upon Blaud's pill. She rapidly improved, and is to-day a buxom woman of 30 , perfectly well. A blood examination in this instance would certainly have shown me the error of my diagnosis; and would have saved the family much unnecessary worry; but I would not have had their unmerited praise, for so great is the faith of the fanily, because this patient was apparently cured of a fatal malady, that to this day they believe I permanently cure $a$ the patient of heart disease. Unfortunately for patients generally, no such happy ending occurs until a proper diagnosis is made, which can be reached by a blood examination alone.

In cases of chlorosis perhaps the most important use of blood examination is to determine when the patient is recovered. I have frequently seen cases of chlorosis which had.all of the typical symptoms return after one or two weeks of proper treatment and declare themselves entirely well. True, all symptoms were much less, but a blood examination showed but little change in the hemoglobin or in the number of corpuscles. Doul,rless many of the cases which have dragged on almost indefinitely in the hands of other physicians have done 
so because of the failure to be accurate, by carefully examining the degree of hemoglobin and the number of blood-cells in all cases before they are discharged. No case of chlorosis should be discharged until the hemoglobin reaches a degree of 80 or 85 per cent., and the corpuscles $4,000,000$ to $5,000,000$.

For years malaria has been made the scapegoat for all conditions of the character of which we may be uncertain. Every patient with an intermittent fever associated with malaise of uncertain origin-especially if such a patient resided in a malarial district-was said to be suffering from malaria. Malaria is to-day one of the few diseases that can be positively diagnosed. I believe that no case should be diagnosed as malaria unless the plasmodium malariæ has been found in the blood. Examinations for this organism need a microscope and the knowledge how to use it. Unfortunately the blood is not frequently submitted to this test. If this were done, beginning cases of tuberculosis and intermittent fevers in pneumonia would be much less frequently called malaria.

During the last winter the following case came under my care: A lad of 8 was suddenly seized at midnight with a chill, followed with temperature rising to 104 . By noon the next day the temperature was normal. The next day at 1 p. m. there was a chill, followed again by a rise of temperature to 104 , which declined to normal by midnight. This was repcated the third day. A careful examination of the blood showed the absence of the malarial organism and the presence of a moderate leucocytosis. By the fourth day an undoubted consolidation of the lung occurred and the case followed the ordinary course of pneumonia in childhood. Examination of the blood in this case absolutely excluded malaria and directed attention to the lungs, which gave no sign whatever until the fourth day.

In cases of anemia of obscure cause, frequently a simple examination of the blood with a microscope will make at least a suggestive diagnosis. Egregious errors in diagnosis of cases of leukemia and pernicious allemia may be made by a very simple view of the blood under the microscope, as exemplified in the following cases; I was requested to see a case which had been treated for two years, first for tuberculosis, and later for malaria, in the first instance, because of increasing pallor and loss of strength, in the latter, because of an enormously enlarged spleen. A simple microscopic slide of blood showed a large increase of the white cells; a later count showed 170,000 per c.c. It is true that neither of the diagnoses made in this case was justifiable, but they would never have been entertained had the gentlemenboth excellent and wise practitioners of medicine-cultivated the habit of examination of the blood in obscure cases.

Another case came to me at the University of Pennsylvania with a diagnosis of cancer of the stomach, made on account of rapid failure of strength with severe gastric symptoms, together with increasing pallor. Physical examination showed no signs which would warrant a diagnosis of malignant disease; and blood examination showed a decrease of the hemoglobin to 20 per cent., with a marked diminution of the red-cells to 900,000. A slide showed marked poikilocytosis, and later a stained specimen showed the marked histological characteristics of pernicious anemia. Here, too, a blood examination, not necessarily extensive, would have saved the patient the absolute hopelessness occasioned by a diagnosis of malignant disease, and the doctor an avoidable error. Widal's serum reaction is to-day practiced by nearly all; a combination of this test with that of examination of the blood for the malarial organism will serve to positively determine between typhoid and malaria, a combination of the two diseases, or neither.

It is known that a count of the leucocytes will frequently decide between a specific fever such as typhoid and some inflammatory condition. The following case is an example of this fact: Miss $P$. was seen in consultation, with the following symptoms: Four years ago the patient suffered with typhoid fever, and at that time the attending physician noticed disease of the mitral valve. Four weeks before I saw her, she was seized with an intermittent fever, with occasional severe chills followed by an exacerbation of the fever and exhausting sweats. Physical examination showed an enormously dilated heart as the result of a double mitral disease, with no other physical signs. The fever was intermittent in type with marked daily remissions, though a true intermittency did not exist. The fever was not typical of mallaria, which was the diagnosis that had been made. Examination of the blood by the board of health showed Widal's serum test to be positive. On account of the previous undoubted attack of typhoid fever, however, this was of little value. Examination for the plasmodium, repeated, showed its absence, and proved the case not malarial. The blood-count showed 40 per cent. hemoglobin; 3,000,000 red cells and 20,000 white cells, a most important factor in connection with valvular disease of the heart, in determining the diagnosis of an ulcerative endocarditis. The diagnosis was confirmed by the subsequent behavior of the case.

It is a well-known fact that in certain cases where the physical signs in the lungs are delayed, an increasing leucocytosis will help to determine the case as one of pneumonia, much to the benefit of the patient and the comfort of the physician.

It will be seen that I have not referred to the staining of the blood and the invaluable and often absolutely necessary steps toward the full study of the case. I have avoided this because such steps need a special training and familiarity with methods not always practicable in everyday practice. But if the preliminary and frequently entirely satisfactory steps be taken, then almost invariably some one can be found who will complete the examinations. The apparatus to be used in this means of diagnosis which I advocate are a microscope, slides, coverslips, hemoglobinometer and hemocytometer.

By the use of a microscope and a slide of fresh blood, one may observe, 1 , whether the leucocytes are largely increased in number; 2 , whether the red cells form properly in rouleaux, and whether they are of odd shapes; 3 , the presence or absence of malarial organism. The first two observations may be made with an ordinary $1 / 6$ objective. The last requires a higher power, preferably an oil-immersion. I have constantly at hand a case containing the latter two instruments, together with diluents and a most necessary rubber bulb. It takes but a few minutes-fifteen-at a patient's home to get the blood properly. The counting and examination can be done at leisure at home. Then, too, there is no undue display of instruments in the presence of the patients. The fresh blood may be preserved for several hours by using oil of cedar run under the edge of the slide. The diluted blood in the tubes can be easily preserved by putting an ordinary gum band lengthwise about them.

In conclusion, let me insist that this most valuable diagnostic measure is practicable for the ordinary, everyday physician. It will enable him to avoid many errors 
in diagnosis. It will help him to take a deeper, more scientific interest in his cases.

DISCUSSION ON PAPERS OF DRS. CAMAC AND FUSSELL.

Dr. William OsLer, Baltimore-I have been for many years impressed with the value of the clinical laboratory and the small laboratory in the wards; we can not any longer do without either; they are really associated; they are as essential to the proper equipment of the hospital as the internes. They are to the physician just as the knife and scalpel are to the surgeon. I was glad that Dr. Camac referred particularly to the need of the clinical laboratory in the outdoor department of the dispensaries, for, after all, it is in the dispensaries that cases are most frequently not only diagnosed, but where they come for diagnosis. A very much larger number of patients would receive good treatment if they received proper diagnosis; and so I think the dispensary should have the aid of a small elinical laboratory. An interesting point is that the equipment need not be elaborate. A small equipment will suffice. An important and essential equipment is in the brain of the dispensary physician and his assistants.

I am particularly glad to hear Dr. Fussell and to see his bag. I wrould rather see his bag than see Dr. Fussell. The doctor can be seen at any time but not his bag. That is the sort of practitioner into whose hands I should like to fall if I had a case of ulcerative endocarditis; that is the sort of a man who not only has a microscope but knows how to use it. The average practitioner needs a microscope, needs it with him in his bag.

\section{Therapeutics.}

Lasting Anesthesia.

Iasting anesthesia can be produced by spraying ethyl chlorid over a surface previously moistened with a concentrated watery solution of cocain. Cocainized ethyl chlorid has been employed for opening abscesses, etc.-Bardet: Med. Rec.

\section{Pruritus Senilis and Its Treatment.}

Parisot, in the Revue méd. de L'E'st, associates pruritus senilis with an autointoxication which has its origin in the intestinal tract and is accompanied by the incompetency of the kidneys present in old age. The blood is thus saturated with poisons, which produce a direct irritation of the nerveterminals. In old age the liver, in an autotoxic sense, does insufficient work, the activity of the kidneys is diminished and, on account of retardation of digestion, the intestines always contain large quantities of fecal matter. The conclusion is that senile pruritus is a toxin-urodemia. The senile changes in the skin produce only a local pruritus, never a general condition. The treatment which the author reeommends consists of benzoic acid, naphthol in daily doses of thirty grains, careful regulation of the bowels and a milk eure.Albany Medical Annals.

\section{Reflex Coughs of Children.}

The editor of Pediatrics, referring to the two principal varieties, quotes MacCoy to the effect that hacking nightcoughs of children are mostly due to nasopharyngeal obstruction, with mechanical irritation by retained secretions. The other class, a paroxysmal hacking cough, described by Dr. Francis Warner, of London, oceurs in emaciated children with poor appetites, but who have a normal temperature and appar ently healthy lungs, and is attributed by him not to peripheral irritations, as of intestinal worms, but to unbalanced central merve-action. This opinion, somewhat at variance with that generally held by the profession at large, is based on the examination of 22,000 school children.

\section{Acute Intestinal Catarrh.}

Professor Ewald prescribes in obstinate acute intestinal catarrh:

R. Resoreini $\ldots \ldots \ldots \ldots \ldots \ldots \ldots \ldots \ldots$ gr. 1xxy Bismuthi salicylatis.

Tanningen, $\overline{\bar{a}} \mathbf{a} \ldots \ldots \ldots \ldots \ldots \ldots \ldots \ldots \ldots \ldots \ldots \ldots$

Sodium carbonate...............

M. ft. pul. Sig. Small teaspoonful every two hours.Georgia Med. and Surg. Jour.

\section{Urticaria.}

Mr. Skinner, pharmacist to the Great Northern Hospital, recommends the following formula for allaying the itching, burning sensation of urticaria:

$\mathbf{R}$. Liquoris hammamelidis............. $\boldsymbol{\xi}_{\mathbf{i i}}$ Salis maris $\ldots \ldots \ldots \ldots \ldots \ldots \ldots \ldots \ldots \ldots$ ofss Aq. $\operatorname{dest...\ldots \ldots \ldots \ldots \ldots \ldots \ldots \ldots \ldots \ldots \ldots ,~} \mathbf{0 i}$

M. Sig. To be applied freely.

He also speaks highly of the following cold cream:

R. Adipis benzoinat ................ Ceræ albæ $\ldots \ldots \ldots \ldots \ldots \ldots \ldots \ldots \ldots$ ₹ Cetacei $\ldots \ldots \ldots \ldots \ldots \ldots \ldots \ldots \ldots \ldots \ldots \ldots \ldots \ldots, \mathbf{3}_{\mathbf{3}}$

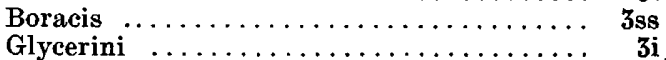
M. Aq. coloniensis...............

\section{-Therapeutic Gazette.}

Migraine Accompanying Anemia.

R. Ext. cannabis indicæ.............. Ac. arseniosi.................... 1 1/60 Ferri pulv......................... i M. Ft. pil. No. 1. Sig. One pill three times a day.

Or:

R. Ext. cannabis indicæ................gr. 1/6 Pulv. digitalis.................... Ferri lactatis....................... ii M. ft. pil. No. i. Sig. One pill three times a day after meals. -Med. Standard.

Inhalation of Balsam in Catarrh of Upper Air-Passages.

In laryngitis and catarrhal bronchitis, a few drops of the following mixture is to be placed in a caraffe-shaped bottle and heated gently over the flame of a spirit lamp. When the bulb becomes filled with the vapors they are drawn by the patient through the nose by means of a tube with a flaring end.

H. Eucalyptol .................... $\mid 25$

Menthol $\ldots \ldots \ldots \ldots \ldots \ldots \ldots \ldots \ldots \ldots \ldots \ldots \ldots$

Terpinol $\ldots \ldots \ldots \ldots \ldots \ldots \ldots \ldots \ldots, 2$

Essence of pine ................ 07

M. For local use-Kafemann: Med. Kec.

\section{Instant Relief of After-Pains.}

According to Winterburn (Jour. of Obstetrics), in many cases a nice warm meal is better than any medicine; "still, where the pains are exhaustingly severe, $I$ turn to amyl nitrite. This potent drug is a very efficient controller of afterpains, and used cautiously, I see no reason to apprehend harm from it. A neat way of using it is to saturate a small piece of tissue paper with five or six drops, stuff this into a 2-dram vial and request the patient to draw the cork and inhale the odor when she feel the pains coming on. It acts with magical celerity."-Med. Prog.

\section{Painful Dysmenorrhea}

The Progrís Médical, citing Presse Médicale, attributes the following to Dalche:

R. Ergotin $\ldots \ldots \ldots \ldots \ldots \ldots \ldots \ldots \ldots \ldots$, gr. 11/2

Sulphate of quinin.................. $30 / 100$

Powdered digitalis leaves............. 15/100

Powdered coca, q. s.

M. For one pill. Sig. Three to five may be taken daily.N. Y. Med. Jour.

\section{Acute Coryza.}

M. Sanger urges (Therap. Monatschrift.) the importance of adopting the right mode of administering topical agents in this complaint, e. g., medicated snuffs, vapors, etr. They should not be introduced into the nose by forced inspiration. Such forced inspiration increases the existing hyperemia of the nasal nucous membrane, and the swelling, hypersecretion and tendency to sneezing. The best remedial agents are: turpentin, 2 to $\overline{5}$ per cent., alcoholic solution of menthol-camphor, with tannin. cocain and menthol. A mixture of equal parts of peroxid of hydrogen and water is recommended for use in spray. These remedial substances should be introduced into the nose bv mechanical means-sprays, insufflators, etc. Hot aqueous vapor is also useful, and the writer has had excellent results with compressed air, introduced by means of a Politzer ball. In using the last, in order to protect the middle ear, the other nostril and the mouth must be kept open. Relief is also to be obtained by preventing nasal respirations by plugging the nose 